\title{
HÁBITOS ALIMENTARIOS, IMAGEN CORPORAL Y BIENESTAR EMOCIONAL: MENS SANA IN CORPORE SANO
}

\author{
Marcel Elipe-Miravet \\ Grupo Salusex. Dpto. de Psicología Básica, Clínica y Psicobiología \\ Universitat Jaume I, Castelló \\ elipe@uji.es \\ Rafael Ballester-Arnal \\ Grupo Salusex. Dpto. de Psicología Básica, Clínica y Psicobiología \\ Universitat Jaume I, Castelló \\ Jesús Castro-Calvo \\ Grupo Salusex. Dpto. de Psicología Evolutiva y de la Educación \\ Universitat de València. \\ Verónica Cervigón-Carrasco \\ Grupo Salusex. Dpto. de Psicología Básica, Clínica y Psicobiología \\ Universitat Jaume I, Castelló \\ Mar Bisquert-Bover \\ Grupo Salusex. Dpto. de Psicología Básica, Clínica y Psicobiología \\ Universitat Jaume I, Castelló
}

Recepción Artículo: 13 marzo 2020

Admisión Evaluación: 30 marzo 2020

Informe Evaluador 1: 15 abril2020

Informe Evaluador 2: 19 abril 2020

Aprobación Publicación: 20 abril 2020

\begin{abstract}
RESUMEN
Ya desde la época de la antigua Roma se afirmaba que el ejercicio físico, sumado a una buena alimentación, eran la clave para el bienestar mental. Aunque a día de hoy la premisa sigue siendo válida, muchos jóvenes parecen no tenerlo claro, pues el sobrepeso es un problema de salud pública en este subgrupo poblacional. El objetivo es conocer los hábitos alimentarios de la población universitaria, viendo cómo estos se relacionan con su bienestar corporal y emocional. Se hipotetiza que quienes tengan peores hábitos alimentarios padecerán un mayor malestar con su imagen corporal, de la misma forma que su bienestar mental se verá mermado. La muestra está formada por un total de 600 sujetos universitarios (300 mujeres y 300 hombres). Los participantes rellenaron el Cuestionario de Estilo de Vida y Salud (Giménez-García \& Ballester-Arnal, 2017), escogiendo para este trabajo aquellos ítems relacionados con la alimentación, la imagen corporal y la salud mental. Aquellas personas que cuidan más su alimentación gozan de una mayor satisfacción con su cuerpo ( ${ }^{2}=45.86 ; p<.001$ ). Al mismo tiempo, los jóvenes que están más orgullosos de la imagen corporal reportan un mayor bienestar emocional $(2=35.02 ; p<.001)$. Aquellas personas que se autodefinen como gruesas reportan peor salud mental que las delgadas 0 las que tienen un peso dentro de los parámetros de la normalidad ( ${ }^{2}=17.26 ; p=.002$ ), Ilegando a cuidar significativamente menos su alimentación ( ${ }^{2}=23.34 ; p<.001$ ). El bienestar emocional, la imagen corporal y el
\end{abstract}




\section{HÁBITOS ALIMENTARIOS, IMAGEN CORPORAL Y BIENESTAR EMOCIONAL: MENS SANA IN CORPORE SANO}

cuidado de la alimentación están estrechamente relacionados. Una dieta equilibrada no solo mejora la salud física, sino que también incrementa la salud mental. Desde los ámbitos de la salud y la educación se ha de concienciar a los jóvenes para mejorar la alimentación. De esta forma se mejorará tanto la satisfacción corporal como su bienestar emocional.

Palabras clave: bienestar emocional; hábitos de alimentación; salud mental; satisfacción corporal

\section{ABSTRACT}

Eating habits, body image and emotional well-being: Mens Sana in Corpore Sano. From the time of ancient Rome, it was stated that physical exercise, added to a good diet, were the key to people's mental well-being. Although the premise is still valid nowadays, young people do not seem to understand it, as being overweight is a real public health problem in this population subgroup. The objective is to know the university population eating habits, seeing how these are related to their body and their emotional well-being. It is hypothesized that those who have worse eating habits will suffer greater discomfort with their body image, in the same way that their mental well-being will be diminished. 600 university students ( 300 women and 300 men) filled out the Lifestyle and Health Questionnaire (Giménez-García \& Ballester-Arnal, 2017). For this work where chosen those items related to food, body image and mental health. People who take care of their diet have a greater satisfaction with their body $\left({ }^{2}=45.86 ; p<.001\right)$. At the same time, people who are proud of their body image report a greater emotional well-being ( $\left.{ }^{2}=35.02 ; p<.001\right)$. Youth who define themselves as a fat person report worse mental health than thin or normal-weight people $\left({ }^{2}=17.26 ; p=.002\right)$, taking significantly less care of their diet $\left({ }^{2}=23.34 ; p<.001\right)$. Emotional well-being, body image and diet care are closely related. A balanced diet not only improves physical health, but also increases mental health. Health and education professionals should encourage young people to improve their diet. This will improve both body satisfaction and emotional well-being.

Keywords: emotional health; well-being; eating habits; mental health; body satisfaction

\section{INTRODUCCIÓN}

La alimentación es uno de los aspectos más importantes de los seres humanos. De ésta depende en gran parte que tengamos una buena condición física y una buena salud mental. El hecho de cuidar poco la alimentación incide en la aparición de problemas de salud en la población general, como el sobrepeso o la obesidad, enfermedades que, a su vez, aumentan la probabilidad de padecer problemas psicológicos como la depresión (Durso \& Latner, 2008; Higuera-Hernández et al., 2018) o la ansiedad (Durso \& Latner, 2008; Mäkinen et al., 2014), y favorecen factores de riesgo como el aislamiento social (Uzunian \& Vitalle, 2015). Uno de los colectivos que más suelen descuidar la alimentación son los jóvenes universitarios (Farhat, lannotti, \& Simons-Morton, 2010). El estrés diario que sufren por los estudios, el hecho de que muchos de ellos vivan fuera de casa compartiendo piso con otros estudiantes, o la agitada vida social que tienen a esas edades hacen que se descuide con demasiada frecuencia su alimentación, llegando a ser deficiente en muchos casos (Alakaam, Castellanos, Bodzio, \& Harrison, 2015; Stockton \& Baker, 2013; Zamsad, Banik, \& Ghosh, 2019). Diversos estudios demuestran que las consecuencias psicológicas de este mal hábito alimentario son más severas en los jóvenes en general (Akbaraly, Sabia, Shipley, Batty, \& Kivimaki, 2013; Klaczynski, Goold, \& Mudry, 2004; Ramón-Arbués et al., 2019) y en jóvenes universitarios en particular (Finato et al., 2013; Moehlecke, Blume, Cureau, Kieling, \& Schaan, 2020).

Las consecuencias de una mala alimentación también afectan directamente a la autoestima de los jóvenes, Ios cuales, además, tienen que lidiar con los estereotipos corporales generados por el grupo de amigos o la propia sociedad (Hovrud, Simons, \& Simons, 2019). Esta última, junto con las tendencias de moda que imperan desde hace años, influyen en gran medida en la actitud de los jóvenes, los cuales siguen dietas muy estrictas con tal de cumplir con los estereotipos que imperan. Es decir, el sobrepeso está mal visto y castigado y, por lo tanto, muchos jóvenes se afanan en conseguir unos cuerpos extremadamente delgados. Muchos jóvenes con ligero sobrepeso se autoimponen la obligación de consumir drogas como alcohol, hachís o marihuana (Pasch, Nelson, 
Lytle, Moe, \& Perry, 2008), con la finalidad de mejorar la imagen que emiten de cara al exterior y conseguir, de esta forma, ser aceptados en el grupo de iguales (Zeller, Reiter-Purtill, \& Ramey, 2008). Además, estos consumos suelen ser más elevados que los de otros jóvenes, pues la exposición a estresores por parte de los jóvenes con la autoestima afectada hace que se incremente significativamente el consumo de estas sustancias (Romero, Martínez, \& Carvajal, 2007). Esto provoca que se lleven a cabo de forma habitual una serie de consumos abusivos de sustancias, además de potenciar la realización de conductas de riesgo innecesarias, con la única finalidad de ser aceptado en la sociedad y sobre todo en su grupo de iguales (Kauffman et al., 2018).

Por lo tanto, los objetivos de este trabajo son conocer cuáles son los hábitos de alimentación que tienen los jóvenes universitarios, la relación entre el cuidado de la alimentación, la percepción de la imagen corporal y la satisfacción que tienen con su propio cuerpo y, por último, si estas dos últimas variables afectan a la salud mental percibida.

\section{MÉTODO}

\section{PARTICIPANTES}

La muestra estaba compuesta por un total de 600 estudiantes (300 mujeres y 300 hombres) de la Universidad Jaume I con edades comprendidas entre 18 y 22 años. El 91\% de los estudiantes eran españoles, el 5\%, latinoamericano, y el $4 \%$ restante, de países de la región europea. Del total, un $29.9 \%$ eran estudiantes de la rama de las ciencias humanas y sociales, un $22 \%$ de ciencias económicas y jurídicas, un $21 \%$ de ciencias de la salud, y un $25.4 \%$ de ciencias tecnológicas y experimentales. Finalmente, un $1.7 \%$ de los encuestados eran estudiantes de doctorado, los cuales pertenecían a las 4 ramas mencionadas anteriormente.

\section{Instrumento}

Para la recogida de los datos se administró el Cuestionario de Estilo de Vida y Salud (Giménez-García \& Ballester-Arnal, 2017) compuesto por 15 bloques relacionados con hábitos de salud. Para los objetivos del presente trabajo, se seleccionó un total de 7 ítems:

- Dos ítems sobre alimentación, en concreto, frecuencia de consumo de alimentos (varias veces al día, una vez al día, varias veces a la semana, varias veces al mes) y cuidado de la alimentación (nada, poco, bastante, mucho), evaluados ambos con escala Likert.

- Dos ítems sobre datos antropométricos, como son el peso y la talla, ambos de formato abierto.

- Dos ítems sobre el aspecto físico, la satisfacción con el cuerpo (nada, poco, bastante, mucho) y cómo se consideraban físicamente (delgados, normales o gruesos), ambos ítems en escala Likert.

- Un ítem que evaluó la salud mental percibida (mala, regular o buena) en escala Likert.

\section{Procedimiento}

Los datos utilizados en esta investigación fueron recogidos durante el curso académico 2018/2019 con la finalidad de realizar un estudio sobre el estado de salud de la comunidad universitaria. Este estudio se enmarca entre las acciones del programa universitario UJI Hábitat Saludable y fue aprobado por la Comisión de Ética de la Universitat Jaume I de Castellón. La forma de administración del cuestionario fue mediante vía telemática y para la difusión se utilizó el correo institucional. En la plataforma de evaluación se proporcionó al alumnado una descripción de los objetivos del estudio y la duración aproximada de la cumplimentación. Del mismo modo, se les aseguró el cumplimiento de las medidas de protección de datos de la LOPD y se solicitó el consentimiento informado.

\section{Análisis estadísticos}

Los datos fueron almacenados y analizados en el programa estadístico SPSS (versión 25). Se realizaron análisis descriptivos para estudiar los hábitos alimentarios y los datos relacionados con la salud de los participantes. 


\section{HÁBITOS ALIMENTARIOS, IMAGEN CORPORAL Y BIENESTAR EMOCIONAL: MENS SANA IN CORPORE SANO}

También se realizaron análisis de la varianza para ver si había diferencias de consumo entre distintos alimentos en función del Índice de Masa Corporal (IMC) de los participantes. Posteriormente, se realizaron contrastes Chicuadrado para ver si la satisfacción con el propio cuerpo y la imagen corporal percibida guardan relación con el cuidado de la alimentación. Finalmente, se utilizó el mismo análisis para ver si la satisfacción corporal y la imagen corporal percibida se relacionan con la salud mental percibida.

\section{RESULTADOS}

1. Cuidado y hábitos de alimentación informados y datos antropométricos

Un 30.2\% de los estudiantes universitarios informó que cuidaba muy poco sus hábitos alimentarios, un 55\% Ios cuidaba bastante y un $14.8 \%$ los cuidaba mucho. En concreto, prácticamente todos los jóvenes realizaban la comida y la cena (un $97.5 \%$ en ambas), porcentaje que disminuye en el desayuno (88.2\%), y sobre todo en el almuerzo (67.8\%) y la merienda (67.2\%).

En cuanto al IMC, un $6.5 \%$ estaba comprendido en el rango de delgadez, un $74.9 \%$ de los participantes se encontraban dentro del rango de la normalidad, un 16.2\% tenía sobrepeso y, finalmente, un 2.4\% padecía obesidad.

Por otra parte, como se puede observar en la Tabla 1, Ios alimentos más perjudiciales para la salud, como los dulces, refrescos o las comidas rápidas, son los que menos se consumían de forma frecuente por la muestra del estudio. Por otro lado, aquellos alimentos que son considerados como esenciales en el día a día tienen porcentajes más altos en su consumo diario, como son las frutas y verduras, los lácteos o los productos cárnicos. En cuanto a los porcentajes de algunos alimentos como son las legumbres o pescados, su consumo diario/semanal es más bajo que el de los alimentos considerados como obligatoriamente diarios, a pesar de que estos también son considerados esenciales en la dieta mediterránea.

\section{Tabla 1}

Porcentajes de consumo de distintos alimentos $(n=600)$

\begin{tabular}{llllll}
\hline & $\begin{array}{l}\text { Casi } \\
\text { Nunca }\end{array}$ & $\begin{array}{l}1-4 \\
\text { veces } \\
\text { Mes }\end{array}$ & $\begin{array}{l}\text { Varias } \\
\text { veces a la } \\
\text { Semana }\end{array}$ & $\begin{array}{l}\text { Diariamente } \\
\text { Varias } \\
\text { veces al } \\
\text { Día }\end{array}$ \\
\hline $\begin{array}{l}\text { Bollería } \\
\text { Precocinado/Comida }\end{array}$ & $37.5 \%$ & $29.6 \%$ & $23.1 \%$ & $8.1 \%$ & $1.7 \%$ \\
$\begin{array}{l}\text { Rápida } \\
\text { Frutas y Verduras }\end{array}$ & $3.5 \%$ & $4.4 \%$ & $20.9 \%$ & $19.2 \%$ & $51.2 \%$ \\
Lácteos & $4.9 \%$ & $6.7 \%$ & $14.4 \%$ & $37.8 \%$ & $36.2 \%$ \\
Refrescos & $34.7 \%$ & $34.9 \%$ & $22.1 \%$ & $5.9 \%$ & $2.3 \%$ \\
Carne & $6.4 \%$ & $3.5 \%$ & $52.6 \%$ & $28.2 \%$ & $9.2 \%$ \\
Pescados & $10.9 \%$ & $22 \%$ & $54.1 \%$ & $11 \%$ & $2 \%$ \\
Legumbres & $6.8 \%$ & $24.7 \%$ & $55.5 \%$ & $10 \%$ & $3 \%$ \\
\hline
\end{tabular}

Finalmente, se cotejó si el IMC de las personas estaba relacionado con las comidas que realizaban al día y por la frecuencia de consumo de los alimentos que aparecen en la Tabla

1. Para ello se realizaron pruebas ANOVA. Solamente se obtuvieron diferencias estadísticamente significativas en la frecuencia de consumo de refrescos, teniendo un mayor IMC aquellos que consumen refrescos varias veces a la semana, en comparación con los que consumen refrescos de forma esporádica ( $F=9.148 ; p<.001)$.

2. Relación entre cuidado de la alimentación informado y la imagen corporal percibida y la satisfacción corporal

Se quiso constatar si había diferencias en el cuidado de la alimentación en función de la imagen corporal percibida. Como puede observarse en la Tabla 2, un porcentaje más elevado de las personas que se consideran 
gruesas, ha indicado que no cuida nada o poco su alimentación con respecto a las que se consideran normales 0 delgadas. Si atendemos a los niveles de bastante y mucho cuidado de alimentación, hay un mayor porcentaje de las personas consideradas delgadas (75.4\%), seguidas de las que se encuentran dentro del rango de la normalidad (71.5\%) y las gruesas en último lugar (54.3\%). Se observan diferencias estadísticamente significativas $\left({ }^{2}=23.34 ; p<.001\right)$.

Tabla 2

Cuidado de la alimentación en función de la imagen corporal percibida ( $n=600)$

\begin{tabular}{lccc}
\hline & \multicolumn{3}{c}{ ¿Físicamente, cómo te consideras? } \\
\cline { 2 - 4 } Cuidado de la alimentación & Grueso/a & Delgado/a & Normal \\
\hline Nada & $7.2 \%$ & $0 \%$ & $1.5 \%$ \\
Poco & $38.6 \%$ & $24.6 \%$ & $27 \%$ \\
Bastante & $38.6 \%$ & $61.1 \%$ & $56.6 \%$ \\
Mucho & $15.7 \%$ & $14.3 \%$ & $14.9 \%$ \\
\hline
\end{tabular}

Por otra parte, se evaluó si la satisfacción corporal se relaciona con el cuidado de la alimentación. Los resultados demuestran que un mayor porcentaje de las personas que informan que cuidan bastante y mucho su alimentación, también indican estar bastante y muy satisfechas con su cuerpo. Y, el 50\% de las personas que informan que no cuidan nada su alimentación se muestra poco o nada satisfechas con su cuerpo. Si observamos los porcentajes en cada intersección nos damos cuenta de la clara relación entre ambas variables, dado que los mayores porcentajes se encuentran en la coincidencia entre el nivel de cuidado de la alimentación y el de la satisfacción corporal (nada-nada, poco-poco....). Eso no significa evidentemente que no haya importantes porcentajes de personas que aun cuidando nada o poco su alimentación estén bastante satisfechos con su cuerpo y viceversa. Se observan diferencias estadísticamente significativas ( $\left.{ }^{2}=45.86 ; p<.001\right)$.

Tabla 3

Satisfacción corporal en función del cuidado de la alimentación ( $n=600)$

\begin{tabular}{lcccc}
\hline & \multicolumn{4}{c}{ Cuidado de la alimentación } \\
\cline { 2 - 5 } Satisfacción corporal & Nada & Poco & Bastante & Mucho \\
\hline Nada & $16,7 \%$ & $1,2 \%$ & $2,1 \%$ & $3,4 \%$ \\
Poco & $33,3 \%$ & $41,7 \%$ & $22,5 \%$ & $23,9 \%$ \\
Bastante & $41,7 \%$ & $53 \%$ & $68,4 \%$ & $55,7 \%$ \\
Mucho & $8,3 \%$ & $4,2 \%$ & $7 \%$ & $17 \%$ \\
\hline
\end{tabular}

3. Relación entre imagen corporal percibida y satisfacción corporal con nivel de salud mental percibida

Con la finalidad de comprobar si la percepción de la imagen corporal se relaciona con la salud mental de las personas, se realizó una prueba Chi-cuadrado. Los porcentajes de mala salud mental percibida son similares para todas las categorías de imagen corporal, ligeramente superiores en las personas que se consideran delgadas. Por otra parte, el mayor porcentaje de los que informan una salud mental regular se encuentra entre los que se consideran gruesos. Y, por último, los que consideran que tienen un peso normal tiene porcentajes más elevados en la percepción de una buena salud mental, seguidos por los que se consideran delgados y los gruesos (Tabla 4). Estas diferencias son estadísticamente significativas ( ${ }^{2}=17.26 ; p=.002$ ). 


\section{HÁBITOS ALIMENTARIOS, IMAGEN CORPORAL Y BIENESTAR EMOCIONAL: MENS SANA IN CORPORE SANO}

Tabla 4

Salud mental percibida en función de la imagen corporal ( $n=600)$

\begin{tabular}{llll}
\hline & \multicolumn{3}{l}{ ¿Físicamente, cómo te consideras? } \\
\cline { 2 - 4 } Salud mental & Grueso/a & Delgado/a & Normal \\
\hline Mala & $3.6 \%$ & $4.0 \%$ & $3.3 \%$ \\
Regular & $26.5 \%$ & $18.3 \%$ & $10.3 \%$ \\
Buena & $69.9 \%$ & $77.8 \%$ & $86.4 \%$ \\
\hline
\end{tabular}

En cuanto a la satisfacción corporal, los resultados demuestran que los que están más satisfechos con su cuerpo perciben también una mejor salud mental, mientras que los que menos satisfechos están, informan de una peor salud mental ( $\left.{ }^{2}=35.02 ; p<.001\right)$. En la Tabla 5 pueden observarse esas diferencias mediante porcentajes.

Tabla 5

Salud mental percibida en función de la satisfacción corporal $(n=600)$

\begin{tabular}{lllll}
\hline \multirow{2}{*}{ Salud mental } & \multicolumn{3}{l}{ Satisfacción corporal } & \\
\cline { 2 - 5 } & Nada & Poco & Bastante & Mucho \\
\hline Mala & $14.3 \%$ & $3 \%$ & $2.7 \%$ & $8.7 \%$ \\
Regular & $35.7 \%$ & $23.7 \%$ & $10.1 \%$ & $6.5 \%$ \\
Buena & $50 \%$ & $73.4 \%$ & $87.2 \%$ & $84.8 \%$ \\
\hline
\end{tabular}

\section{DISCUSIÓN}

Una de las consecuencias de la vida universitaria es el descuido de la alimentación. Estudios como el de Farhat, lannotti, y Simons-Morton (2010) ya alertaban que los estudiantes que cursan estudios superiores tienen una alimentación más deficiente que el resto de la población. Factores estresantes como los estudios o la extensa vida social hacen que la alimentación y otros hábitos de salud queden en un segundo plano.

En primer lugar, se ha podido comprobar que casi todos los estudiantes universitarios realizan dos comidas fuertes al día, la comida y la cena, y el porcentaje va disminuyendo cuando se trata del desayuno, el almuerzo y la merienda. Además, algunos de ellos complementan estas comidas con algún bocado a media mañana o tarde. En las últimas décadas ha existido mucha controversia en cuanto al número de comidas que se tenían que realizar al día. Actualmente, en lo que los nutricionistas y dietistas parecen estar de acuerdo es que no importa las comidas que se hagan al día, siempre y cuando estas se hagan cuando uno tenga hambre y coma de forma saludable, evitando alimentos altos en grasas saturadas y con altos contenidos de azúcar (Barrios et al., 2015; Paoli, Tinsley, Bianco, \& Moro, 2019).

En cuanto a la frecuencia de consumo de los alimentos, podemos afirmar que el consumo diario de elementos como frutas y verduras o lácteos es bastante bajo, pues menos de la mitad de los jóvenes universitarios de nuestro estudio consume frutas, verduras y lácteos de forma diaria, debiendo ser este consumo mucho más elevado (León-Muñoz et al. (2012). Algo similar ocurre con las proteínas de origen animal. Carnes y pescados han de ser la fuente de proteínas del organismo; sin embargo, su consumo es más bajo del recomendado (Paoli, Tinsley, Bianco, y Moro (2019). Ahora bien, la ingesta de refrescos, comidas precocinadas o bollería industrial es bastante baja.

A pesar de que los hábitos de consumo mencionados anteriormente no son tan buenos como se recomienda, el IMC de los jóvenes es bastante mejor que el de la población adulta española (Hábitos de Vida, 2019), los cuales padecen más obesidad y sobrepeso que nuestra muestra de estudio.

La imagen corporal está altamente relacionada con nuestros hábitos alimentarios. Estos hallazgos que aquí hemos encontrado también han sido observados en estudios como el de Stice, Marti y Durant (2011), quienes vieron que quienes se consideraban que estaban dentro del rango normal de peso cuidaban más su alimentación. 
Es más, la satisfacción con el propio cuerpo también parece influir en lo que se come, aunque la relación también podría ser la inversa. Estudios como el de Oliveira et al. (2020) llegan a la misma conclusión que los resultados aquí encontrados, pues quienes más satisfechos están con su cuerpo, más cuidan su alimentación o viceversa.

En cuanto a verse físicamente por encima del peso normal y padecer peor salud mental, los resultados que hemos encontrado concuerdan con la bibliografía existente (Finato et al., 2013; Moehlecke, Blume, Cureau, Kieling, \& Schaan, 2020). Los estudios al respecto afirman que un gran porcentaje de adolescentes o jóvenes que están por encima de la categoría del IMC considerada como normal padecen emocionalmente más que aquellos sujetos que se encuentra en esa categoría o por debajo. Por lo tanto, los hallazgos de este trabajo en cuanto a disfrutar de una mejor salud mental al cuidar los hábitos de alimentación no son distintos a otros estudios realizados en población general (Nayir et al., 2016).

El estudio, obviamente, cuenta con algunas limitaciones. En primer lugar, sería recomendable tener en cuenta otros factores que puedan influir en las variables aquí analizadas como, por ejemplo, el ejercicio físico. Del mismo modo, sería conveniente aportar una perspectiva de género a este estudio, dado que las mujeres suelen ser las que experimentan más problemas relacionados con la alimentación y la imagen corporal (Tayfur \& Evrensel, 2020).

A pesar de ello, se considera que estos hallazgos suponen un punto de inflexión para el binomio de la educación y la salud. Es fundamental que ambos ámbitos se integren y se incida en la importancia que tiene el estilo de vida, en concreto una adecuada alimentación. Sería importante que los estudiantes universitarios recibieran formación transversal o participaran activamente en acciones de formación informal durante su educación superior. Proyectos como el de la Red Española de Universidades Saludables, en el cual se encuentra la Universitat Jaume I, cumplen con la necesidad de mejorar la salud de la comunidad universitaria. Una adecuada educación en hábitos alimentarios no solo mejoraría la salud general de los estudiantes a corto plazo, sino que contribuirá a la prevención de muchas enfermedades crónica a medio y largo plazo, y ayudará a promocionar la salud mental.

\section{REFERENCIAS BIBLIOGRÁFICAS}

Akbaraly, T., Sabia, S., Shipley, M., Batty, G., \& Kivimaki, M. (2013). Adherence to healthy dietary guidelines and future depressive symptoms: evidence for sex differentials in the Whitehall II study. The American Journal of Clinical Nutrition, 97(2), 419-427. doi: 10.3945/ajcn.112.041582

Alakaam, A. A., Castellanos, D. C., Bodzio, J., \& Harrison, L. (2015). The factors that infl uence dietary habits among international students in United States. Journal of International Students, 5(2), 104-120. Retrieved from https://fi les.eric.ed.gov/fulltext/EJ1060049.pdf

Barrios, R., Navarrete, E. M., García de la Hera, M., González, S., Valera, D., Checa, J. F., Giménez, D., \& Vioque, J. (2015). Una menor adherencia a la dieta mediterránea se asocia a una peor salud auto-percibida en población universitaria. Nutrición Hospitalaria. 31(2), 785-792.

Durso, L. E., \& Latner, J. D. (2008). Understanding self-directed stigma: Development of the Weight Bias Internalization Scale. Obesity, 16, S80-S86. doi: 10.1038/oby.2008.448

Farhat, T., lannotti, R., \& Simons-Morton, B. (2010). Overweight, Obesity, Youth, and Health-Risk Behaviors. American Journal of Preventive Medicine, 38(3), 258-267. doi: 10.1016/j.amepre.2009.10.038

Finato, S., Rech, R., Migon, P., Gavineski, I., Toni, V., \& Halpern, R. (2013). Insatisfação com a imagem corporal em escolares do sexto ano da rede municipal de Caxias do Sul, no Rio Grande do Sul. Revista Paulista de Pediatria, 31(1), 65-70. doi: 10.1590/s0103-05822013000100011

Hábitos de vida. Informe Anual del sistema Nacional de Salud (2019). Recuperado de https://www.mscbs.gob.es/estadEstudios/estadisticas/sisInfSanSNS/tablasEstadisticas/InfAnualSNS2018/C ap.3_HabitosVida.pdf 


\section{HÁBITOS ALIMENTARIOS, IMAGEN CORPORAL Y BIENESTAR EMOCIONAL: MENS SANA IN CORPORE SANO}

Higuera-Hernández, M., Reyes-Cuapio, E., Gutiérrez-Mendoza, M., Rocha, N., Veras, A., \& Budde, H. et al. (2018). Fighting obesity: Non-pharmacological interventions. Clinical Nutrition ESPEN, 25, 50-55. doi: 10.1016/j.cInesp.2018.04.005

Hovrud, L., Simons, R., \& Simons, J. (2019). Cognitive Schemas and Eating Disorder Risk: the Role of Distress Tolerance. International Journal of Cognitive Therapy, 13(1), 54-66. doi: 10.1007/s41811-019-00055-5

Kauffman, B., Bakhshaie, J., Manning, K., Rogers, A., Shepherd, J., \& Zvolensky, M. (2018). The role of emotion dysregulation in the association between subjective social status and eating expectancies among college students. Journal of American College Health, 68(1), 97-103. doi: 10.1080/07448481.2018.1515761

Klaczynski, P. A., Goold, K. W., \& Mudry, J. J. (2004). Culture, obesity stereo-types, self-esteem, and the "thin ideal": A social identity perspective. Journal of Youth and Adolescence, 33, 307-317. doi: 10.1023/B:JOY0.0000032639.71472.19

León-Muñoz, L. M., Guallar-Castillón, P., Graciani, A., López-García, E., Mesas, A.E., Aguilera, M. T. et al. (2012). Adherence to the Mediterranean diet pattern has declined in Spanish adults. Journal of Nutrition, 142, 18431850.

Mäkinen, M., Lindberg, N., Komulainen, E., Puukko-Viertomies, L., Aalberg, V., \& Marttunen, M. (2014). Psychological well-being in adolescents with excess weight. Nordic Journal of Psychiatry, 69(5), 354-363. doi: 10.3109/08039488.2014.986194

Moehlecke, M., Blume, C., Cureau, F., Kieling, C., \& Schaan, B. (2020). Self-perceived body image, dissatisfaction with body weight and nutritional status of Brazilian adolescents: a nationwide study. Jornal de Pediatria, 96(1), 76-83. doi: 10.1016/j.jped.2018.07.006

Nayir, T., Uskun, E., Yürekli, M.V., Devran, H., Çelik, A., \& Okyay, R.A. (2016). Does body image affect quality of life? A population based study. PLoSOne, 11, e0163290. https://doi.org/10.1371/journal.pone.0163290.

Oliveira, N., Coelho, G., Cabral, M., Bezerra, F., Faerstein, E., \& Canella, D. (2020). Association of body image (dis)satisfaction and perception with food consumption according to the NOVA classification: Pró-Saúde Study. Appetite, 144, 104464. doi: 10.1016/j.appet.2019.104464

Paoli, A., Tinsley, G., Bianco, A., \& Moro, T. (2019). The Influence of Meal Frequency and Timing on Health in Humans: The Role of Fasting. Nutrients, 11(4), 719. doi: 10.3390/nu11040719

Pasch, K., Nelson, M., Lytle, L., Moe, S., \& Perry, C. (2008). Adoption of Risk-Related Factors Through Early Adolescence: Associations with Weight Status and Implications for Causal Mechanisms. Journal of Adolescent Health, 43(4), 387-393. doi: 10.1016/j.jadohealth.2008.02.009

Ramón-Arbués, E., Martínez-Abadía, B., Granada -López, J., Echániz-Serrano, E., Pellicer-García, B., Juárez-Vela, R. et al. (2019). Eating behavior and relationships with stress, anxiety, depression and insomnia in university students. Nutrición Hospitalaria, 36(6), 1339-1345. doi: 10.20960/nh.02641

Red Española de Universidades Saludables. Principios, objetivos y estructura de la red [Internet]. 2008 [cited 2020 Apr 9]. Available from: http://servicios.unileon.es/reus/files/2011/07/principios-objetivos-y-estructura-de-la-REUS.pdf

Romero, A., Martínez, D., \& Carvajal, S. (2007). Bicultural Stress and Adolescent Risk Behaviors in a Community Sample of Latinos and Non-Latino European Americans. Ethnicity \& Health, 12(5), 443-463. doi: 10.1080/13557850701616854

Stice, E., Marti, C., \& Durant, S. (2011). Risk factors for onset of eating disorders: Evidence of multiple risk pathways from an 8-year prospective study. Behaviour Research and Therapy, 49(10), 622-627. doi: 10.1016/j.brat.2011.06.009

Stockton, S., \& Baker, D. (2013). College Students' Perceptions of Fast Food Restaurant Menu Items on Health. American Journal of Health Education, 44(2), 74-80. doi: 10.1080/19325037.2013.764242

Tayfur, S., \& Evrensel, A. (2020). Investigation of the relationships between eating attitudes, body image and depression among Turkish university students. Rivista di Psichiatria, 55(2), 90-97. Doi: 10.1708/3333.33023 
Uzunian, L., \& Vitalle, M. (2015). Social habilities: Habilidades sociais: protective factor against eating disorders in adolescents. Ciência \& Saúde Coletiva, 20(11), 3495-3508. doi: 10.1590/1413-812320152011.18362014

Zamsad, M., Banik, S., \& Ghosh, L. (2019). Prevalence of overweight, obesity and abdominal obesity in Bangladeshi university students: A cross-sectional study. Diabetes \& Metabolic Syndrome: Clinical Research \& Reviews, 13(1), 480-483. doi: 10.1016/j.dsx.2018.11.015

Zeller, M., Reiter-Purtill, J., \& Ramey, C. (2008). Negative Peer Perceptions of Obese Children in the Classroom Environment. Obesity, 16(4), 755-762. doi: 10.1038/0by.2008.4 
